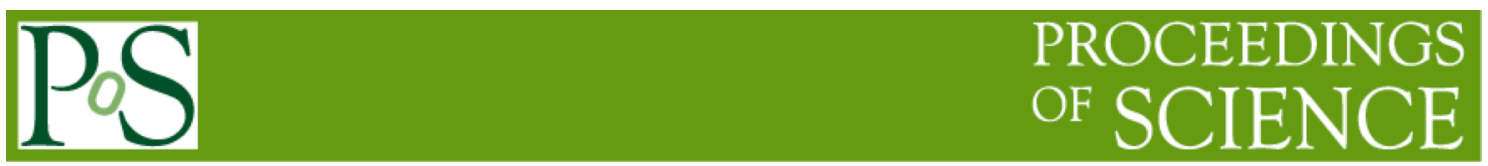

\title{
3D Silicon Detectors
}

\section{Gian-Franco Dalla Betta ${ }^{1}$}

University of Trento and INFN TIFPA

Via Sommarive, 9 - 38123 Povo di Trento, Italy

E-mail: gianfranco.dallabettalunitn.it

\begin{abstract}
Twenty years after their introduction by Sherwood Parker and collaborators, 3D silicon detectors have reached their maturity. The ATLAS IBL represents an important milestone: it is the first application of 3D technology to a High Energy Physics experiment, and it has demonstrated the feasibility of a medium volume production with a relatively good yield. In spite of this significant achievement, the future Phase 2 upgrades of experiments at the HL-LHC are setting very challenging demands in terms of smaller pixel size, reduced sensor thickness, and ultra-high radiation tolerance, calling for the development of a new generation of 3D sensors. This paper reviews the state of the art in 3D detectors, and addresses the next directions of their development in view of the application to HL-LHC. The main design and technological issues are reported, as well as preliminary results from TCAD simulations
\end{abstract}

The $1^{\text {st }}$ INFN Workshop on Future Detectors for LHC

11-13 March 2014

Trento, Italy

\footnotetext{
${ }^{1}$ Speaker
} 


\section{Introduction}

First introduced by Sherwood Parker in 1997 [1], 3D silicon detectors consist of an array of columnar electrodes of both doping types, oriented perpendicularly to the wafer surface and penetrating entirely through the substrate. This unique structure enables to decouple the active sensor thickness from the electrode distance, offering important advantages in terms of low operation voltage, fast time response [2] and high radiation tolerance [3]. Additionally, 3D technology allows for "active edges", i.e., deep trenches heavily doped to act as ohmic terminations of the sensors, able to reduce the insensitive edge region to a few micrometers [4]. Of course this comes at the expense of a more complex and expensive technology [5], due to the use of several non standard techniques, such as Wafer Bonding (WB) [6] and Deep Reactive Ion Etching (DRIE) [7].

In the past few years, very important progress has been made in the development of 3D sensors, passing from the earlier R\&D phase with performance demonstration on a few prototypes (1997-2006) to more systematic studies (2007-2010) and finally to an industrialization phase (2011-2012), which led to the first application of 3D sensors in a High Energy Physics experiment, i.e., the ATLAS Insertable B-Layer (IBL) [8]. Besides confirming the excellent performance and radiation hardness of 3D sensors [9], this important achievement also demonstrated the feasibility of medium volume productions with a relatively good yield ( $\sim 60 \%$ on selected wafers) [10], thus paving the way to using 3D detectors in other experiments planned for the phase 1 (e.g., the ATLAS Forward Physics experiment [11]), and phase 2 upgrades at the High Luminosity LHC (HL-LHC) [12]. In fact, while the former can use the same sensors developed for the IBL [13], the latter are setting more challenging demands in terms of smaller pixel size, reduced sensor thickness, and ultra-high radiation tolerance, that present 3D sensors cannot meet. As a result, a new generation of 3D sensors has to be developed, calling for a dedicated $R \& D$ effort, for which the main requirements, some ideas and preliminary results are presented in this paper.

The paper is organized as follows: in Section 2, the state of the art is reviewed; in Section 3 , the requirements for 3D sensors to be used in phase 2 upgrades at LHC are outlined; possible technological approaches for future 3D sensors are discussed in Section 4, and preliminary layouts and TCAD simulation results are reported in Section 5. Conclusions are drawn in Section 6.

\section{State of the art}

The state of the art in 3D detectors is represented by the IBL sensors, which were developed within the ATLAS 3D Sensor Collaboration with a joint effort of all the processing facilities involved (Stanford, SINTEF, FBK, and CNM), although the final production was carried out only at FBK and CNM [8]. Two different technological approaches currently exist for 3D sensor fabrication: single-sided full 3D with active edges, and double-sided 3D with slim edges.

The single-sided approach was originally proposed at Stanford [5] and is now also available at SINTEF [14]: column etching is performed all through the substrate from the front 
side of the sensor wafer for both types of electrodes, at the same time implementing active ohmic trenches at the edge. Columnar electrodes and trenches are completely filled with polysilicon. The bottom side is oxide bonded to a support wafer. This requires extra steps to attach and remove the support wafer.

The double-sided approach was independently developed by CNM [15] and FBK [16],[17] in slightly different versions: junction columns are etched from the front side, ohmic columns from the back side, without the presence of a support wafer. In the CNM processing, columns do not pass all through the wafer but stop at a short distance from the surface of the opposite side [15]; this was also the case for the first prototypes of FBK sensors [16], but the technology was later modified to allow for passing through columns [17].

Other aspects worth being recalled are the following:

a) single-sided 3D sensors can be made with four-side active edges [4], that instead are not feasible with double-sided 3D sensors, due to the absence of a support wafer. As an alternative, slim edges have been adopted for the IBL with a dead area of about $200 \mu \mathrm{m}$ : while CNM sensors feature a 3D guard ring, surrounded by two rows of ohmic columns, FBK sensors feature a termination only based on ohmic columns [18];

b) in single-sided 3D sensors the substrate bias is applied from the front side, i.e., the same side where bump bonding is made. A possible alternative would require a post-processing treatment after support wafer removal: a hole in the insulating layers on the back side should be etched, and a metal wire bond pad should be deposited, but these extra steps are not fully engineered. On the contrary, in CNM and FBK sensors the substrate bias is applied from the back side, which makes these sensors compatible with standard planar sensors.

c) on wafer electrical tests are of paramount importance to identify bad pixel sensors at an early stage, before bump bonding. To this purpose, Stanford, SINTEF and FBK deposit a temporary metal layer that allows current-voltage tests to be performed in each sensor, whereas in CNM sensors the quality is evaluated by measuring the guard ring current. While the former method was proved to be very accurate, the latter failed to detect defects in the center of the sensors, so it is not reliable enough [19].

From the point of view of the electrical characteristics, one aspect to be carefully considered in view of operation at very large radiation fluences is the possibility to bias 3D sensors at the most appropriate voltage. In this respect, breakdown voltage could be an issue. In case of FBK IBL sensors, the breakdown voltage was relatively low, of the order of $50 \mathrm{~V}$ before irradiation and up to about $160 \mathrm{~V}$ after irradiation. After completing the IBL production, it was then decided to modify the technology and the design to improve the breakdown voltage. In particular, since the weakest point was demonstrated to be the junction column to p-spray junction on the back side, the junction columns were shortened stopping at about $20 \mu \mathrm{m}$ from the opposite surface; moreover, the front side layout was improved [20]. As a result, the breakdown voltage was significantly increased both before and after irradiation [21].

In terms of functional properties, remarkable performances have been demonstrated for the IBL 3D sensors: in particular, they have shown hit reconstruction efficiency $>98 \%$ for $15^{\circ}$ inclined tracks at $160 \mathrm{~V}$ bias after $5 \times 10^{15} \mathrm{n}_{\mathrm{eq}} / \mathrm{cm}^{2}$ [9]. Very encouraging results have also been obtained from the characterization of 3D pixel prototypes compatible with the PSI46 read-out chip [22],[23]. 
An important remark concerns the signal efficiency (SE) of heavily irradiated 3D sensors, which represents a key advantage compared to planar sensors: it was shown that SE essentially depends on geometrical considerations, as expressed by Eq. (1) [24]:

$$
S E=\frac{1}{1+0.6 L \frac{K_{\tau}}{v_{D}} \Phi}
$$

where $\mathrm{L}$ is the inter-electrode spacing, $K_{\tau}$ is the damage constant for the electron effective trapping time, $V_{D}$ is the electron drift velocity, and $\Phi$ is the irradiation fluence. Figure 1 shows a compilation of data relevant to irradiated 3D sensors from different manufacturers, featuring similar values of $\mathrm{L}$, and showing very high signal efficiencies up to high fluences in good agreement with Eq. (1).

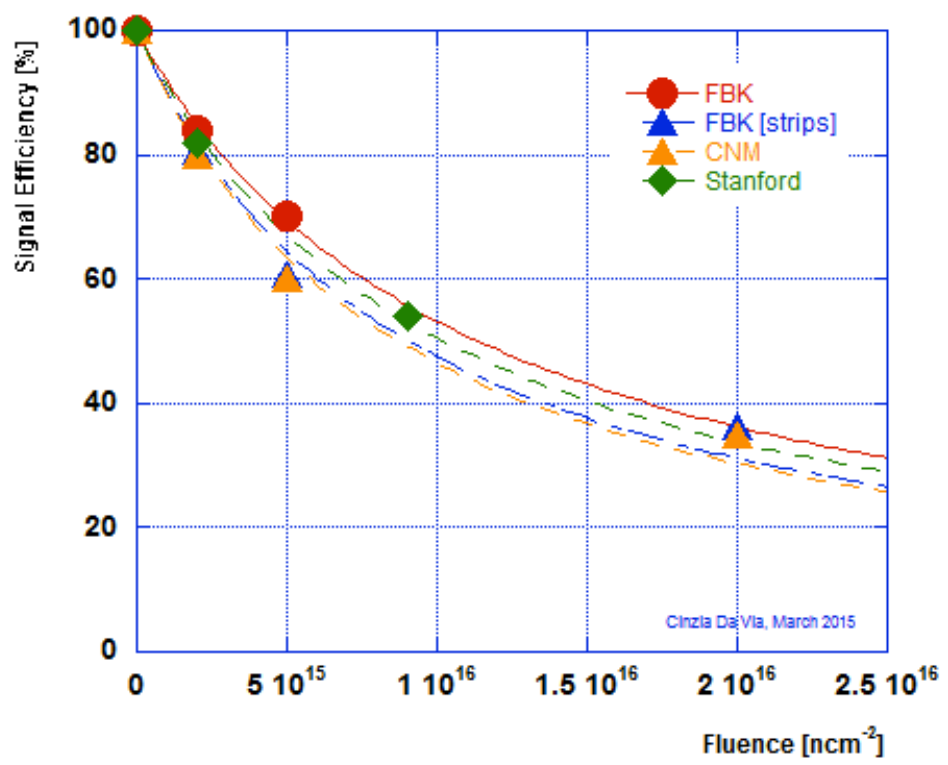

Figure 1 Compilation of signal efficiency in irradiated 3D sensors with fitting curves according to Eq. (1). With respect to the original plot from [10], and including data from FBK pixels [9], CNM strips [25], and Stanford diodes [26], new data from FBK strips are added [27].

\section{Requirements for phase 2 upgrade experiments}

Despite the IBL 3D sensor performances are remarkably good, the phase 2 upgrades at the HL-LHC call for several improvements, in particular in terms of increased radiation hardness (the maximum expected fluence for the inner layer is $2 \times 10^{16} \mathrm{n}_{\mathrm{eq}} / \mathrm{cm}^{2}$ ) and capabilities of an efficient and accurate reconstruction of the tracks produced by charged particles in environments where their spatial density is very high. Occupancy considerations push towards a much higher granularity with respect to the pixels currently installed at the LHC: as an example, pixel dimensions considered by the RD53 Collaboration in the development of future read-out chips in $65 \mathrm{~nm}$ CMOS technology are $50 \times 50 \mu \mathrm{m}^{2}$ and $25 \times 100 \mu \mathrm{m}^{2}$ [28]. Another requirement is to substantially reduce the material budget of each detection layer in order to improve the 
accuracy of the reconstruction of primary vertices and decay. Moreover, a high geometrical efficiency should be pursued to allow for a more hermetic detector design.

The implications of these requirements on 3D sensor design and fabrication technology are manifold and mutually correlated: on one hand, a small inter-electrode spacing (L), comparable with the average carrier drift length due to charge trapping at the maximum considered fluences, is necessary to ensure the radiation hardness. On the other hand, in order to take advantage of the smaller pixel size for a high spatial resolution, the sensor active thickness should be decreased, so as to obtain smaller cluster dimensions. Thinner sensors also contribute to reduce the overall material budget and guarantee optimal track momentum resolution. Further reduction of the material budget can be obtained by implementing active edges (or at least very slim edges), preventing from overlaps between adjacent modules, which are needed to build a hermetic detector, thus reducing the amount of material in those specific regions of solid angle.

Both a small L and a small pixel size lead to a high density of columnar electrodes, with a detrimental effect on the geometrical efficiency (the electrode themselves are not efficient) and on the capacitance (noise). In this respect, electrode diameter should be reduced: assuming the aspect ratio (depth to diameter) attainable with DRIE to be constant, this calls for thinner substrates. In turn, since the electrode capacitance scales with its depth to a large extent, thinner substrates also mean lower noise. Finally, narrower electrodes on thin substrates can ease their (at least) partial filling with poly-Si to obtain some efficiency, at least before irradiation, and to allow for a higher layout flexibility.

From all the above considerations, making 3D sensors on thinner substrates appears the best solution, but there are also two important drawbacks to be considered: the first, obvious one is that thinner substrates also mean lower signals generated by impinging particles, so the thickness cannot be too small and the optimum value should be carefully determined as a compromise of several factors and also taking into account the properties of the read-out chips, and in particular the capability to operate at low thresholds.

The second problem with thinner substrates is their compatibility with the sensor fabrication facility and with the bump bonding process. With reference to FBK, the doublesided technology [17] used for the IBL production proved to offer several advantages in terms of process complexity, overall fabrication times, and sensor assembly within a system, mainly due to the absence of the support wafer. Nevertheless, it is not easy to process a wafer thinner than 200 micron without a support wafer, and this problem is even more critical for 6"- diameter wafers, to which the FBK pilot line was upgraded in 2013. As for the bump bonding, a serious problem with thin wafers is that they are prone to high bowing at the end of the process, whereas low values are normally required (e.g., $<50 \mu \mathrm{m}$ ) in order for the yield of bump bonding to be acceptable. As a result, for thin 3D sensors, single-sided processes with support wafer should be preferred.

\section{Technological approaches to make thin 3D sensors}

With reference to Figure 2, some possible options can be envisioned:

a) according the original approach developed at Stanford, a sensor wafer of the desired thickness is oxide bonded to a support wafer (SOI substrate), which provides a good 
mechanical stability during processing. Both junction (red) and ohmic (blue) columns can be made passing-through, with p-spray on both sides. The main problem is the support wafer removal, for which no fully engineered solution exists to date, and the difficulties, both in terms of layout and assembly, with providing the bias voltage from the front-side (i.e., the same side of the pixels where bump-bonding is performed), unless metal contacts could be made on the back-side after support wafer removal (post-processing).

b) an interesting alternative, compatible only with non-passing-through junction columns (to avoid early breakdown at $\mathrm{n}^{+} / \mathrm{p}^{++}$junctions), is using epitaxial wafers or SiSi direct bonded wafers, where a high resistivity sensor layer is grown/attached on top of a low resistivity substrate; the latter should be finally removed, but a thin portion of it could be left that would still allow a back-side metal to be deposited to apply the bias voltage.

c) As an alternative to support wafer removal as a post processing, option (a) can be followed by local thinning (by DRIE or by wet etching, e.g., by TMAH) of the support wafer from the back-side, while leaving frames of support wafer at the periphery of each sensor to improve its mechanical stability.

d) In case ohmic columns are etched also through the bonding oxide layer, local thinning from the back-side would expose the ohmic columns, thus allowing for bias voltage supply from the back-side by depositing an aluminium layer.

a)

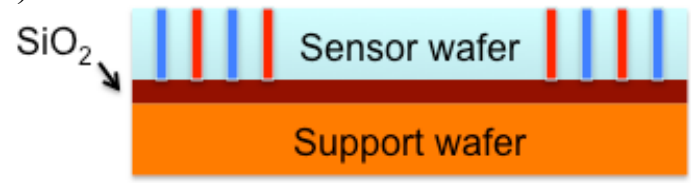

c)

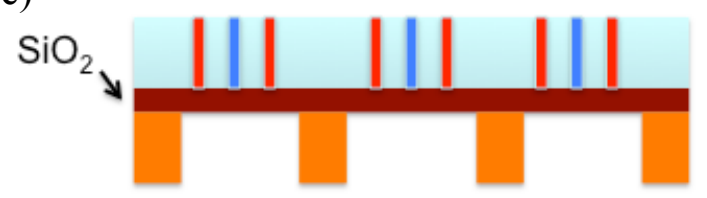

b)

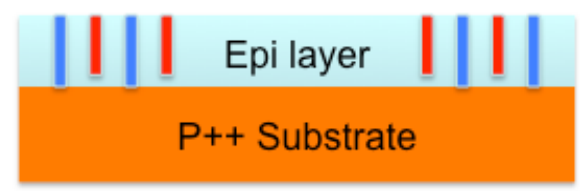

d)

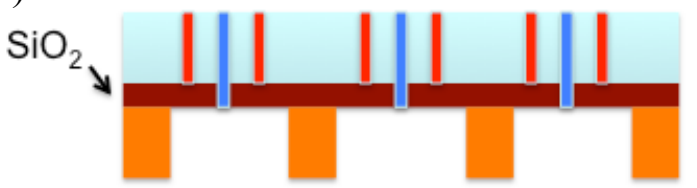

Figure 3 Possible single-sided technological options for thin 3D sensors: (a) SOI wafers "a la Stanford"; (b) epitaxial or Si-Si direct bonded wafer; (c) SOI wafers with back-side local etching; (d) SOI wafers with ohmic columns passing through the oxide and back-side local etching.

All approaches of Fig. 2 use a support wafer, so they are compatible with active edges [4]. As an alternative, slim edges can still be used: those developed for the IBL used a conservative design of $200 \mu \mathrm{m}$ [18], but tests made with more aggressive dicing have shown that $75 \mu \mathrm{m}$ would be sufficient with the present electrode structures, and further improvement are possible either by higher density column designs or different electrode shapes [29].

\section{Preliminary results}

With reference to the technological approach of Fig. 2(b), to be carried out on SiSi direct bonded wafers from Icemos Ltd., preliminary layouts of new 3D pixels compatible with the read-out chips from the RD53 Collaboration have been designed. The considered column 
diameter is $5 \mu \mathrm{m}$ that was proved to be feasible by FBK for DRIE etching down to a depth of about $150 \mu \mathrm{m}$, corresponding to an aspect ratio of almost 30:1.

Figure 3 (left) shows the layouts for the $50 \times 50 \mu \mathrm{m}^{2}$ and the $25 \times 100 \mu \mathrm{m}^{2}$ pixels. The former features one junction $(\mathrm{N})$ column $(1 \mathrm{E})$ at the center of a cell, with an inter-electrode spacing $\mathrm{L}$ of about $36 \mu \mathrm{m}$. In this layout there's room enough to place the bump bonding pad at either side of the junction column. On the contrary, for radiation hardness considerations, the $25 \times 100 \mu \mathrm{m}^{2}$ pixel is designed with two junction columns (2E), with an inter-electrode spacing $\mathrm{L}$ of about $28 \mu \mathrm{m}$. In this case the layout is pretty dense and there are strong constraints on the placement of the bump bonding pad, that would be anyway close both to the junction columns and to the ohmic columns, making this layout not so robust against misalignment problems. In this respect, more flexible designs could benefit from the possibility to place the bonding pads right on top of the junction columns in case they are completely filled with poly-Si.
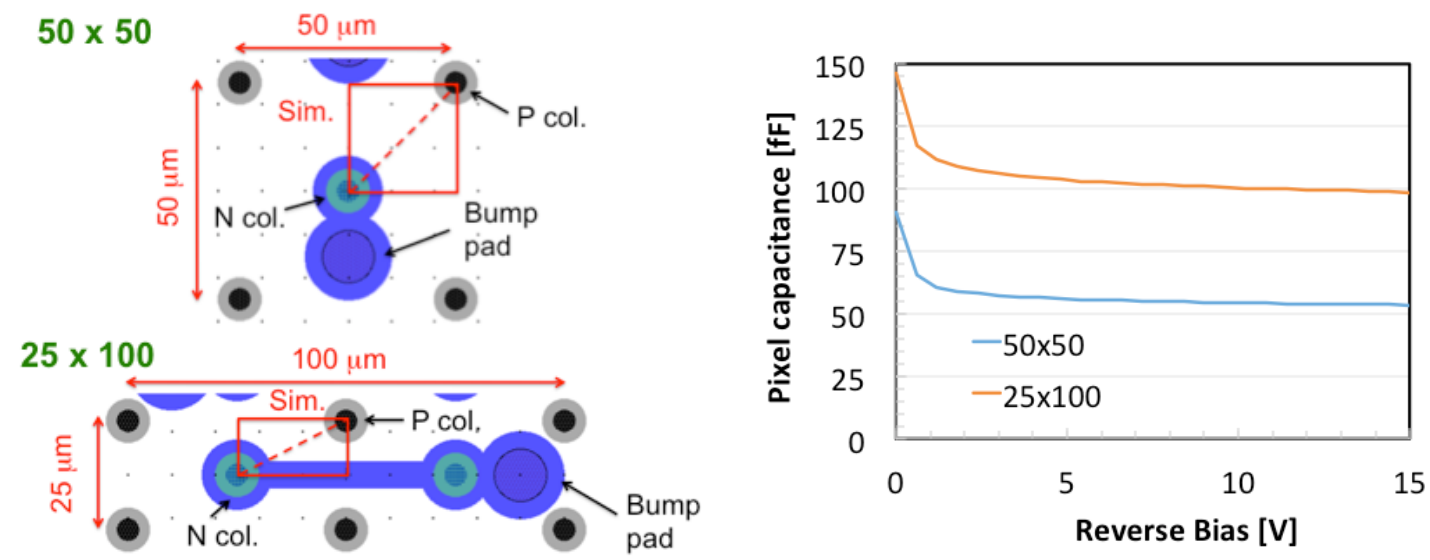

Figure 3 Pixel layout (left) and simulated pixel capacitance for an active thickness of 150 um (right).

The two pixel layouts were evaluated by using TCAD simulations performed with Synopsys Sentaurus. All technological parameters are representative of FBK technology. Simulation domains are those indicated by the red square/rectangle in Figure 3 (left): owing to symmetry considerations, these are the simplest basic cells that can be considered. For capacitance simulations, three-dimensional domains of $150 \mu \mathrm{m}$ thickness and junction column depth of $130 \mu \mathrm{m}$ have been used in order to properly account also for surface effects, that were found to be non negligible in the IBL 3D pixel sensors fabricated at FBK [30]. On the contrary, for charge collection simulations, quasi-2d simulation domains, i.e., 1- $\mu \mathrm{m}$ thick slices, have been considered, incorporating the "Perugia" trap model [31] modified as described in [32] to account for bulk radiation damage.

Figure 3 (right) shows the simulated capacitance versus voltage curves. It can be seen that, owing to the small value of $\mathrm{L}$, full depletion is achieved at very low voltage. The capacitance values are about $50 \mathrm{fF}$ for the $50 \times 50 \mu \mathrm{m}^{2}$ pixel and about $100 \mathrm{fF}$ for the $25 \times 100 \mu \mathrm{m}^{2}$ one: this is mainly due to the $1 \mathrm{E}$ configuration of the former and the $2 \mathrm{E}$ configuration of the latter. Both values include all contributions intrinsic to the $3 \mathrm{D}$ sensor, but not that from the bumps, that 
might add a few tens of $\mathrm{fF}$. In this respect, it is clear that the $25 \times 100 \mu \mathrm{m}^{2}$ pixel is more critical with a total capacitance that would exceed the $100 \mathrm{fF}$ limit set by the RD53 Collaboration [28].

As far as the signal efficiency is concerned, simulations of the two pixels at different irradiation fluences are reported in Fig. 4. Owing to the smaller value of $\mathrm{L}$, the superior performance of the $25 \times 100 \mu \mathrm{m}^{2}$ pixel is evident both in terms the maximum values of the signal efficiency and of the voltage necessary to reach them. In particular, with reference to the maximum fluence, it can be seen that the $25 \times 100 \mu \mathrm{m}^{2}$ pixel features a signal efficiency of more than $50 \%$ already at $120 \mathrm{~V}$, whereas the signal efficiency of the $50 \times 50 \mu \mathrm{m}^{2}$ pixel slightly exceeds $30 \%$ (and is not yet saturated) at $250 \mathrm{~V}$.

Nevertheless, it should be mentioned that the considered radiation damage model was validated up to irradiation fluences in the order of $1 \times 10^{15} \mathrm{n}_{\mathrm{eq}} / \mathrm{cm}^{2}$ and tends to underestimate the signal efficiency at higher fluences. As an example, the same model provided an excellent agreement with experimental data for 3D sensors from FBK irradiated with protons at $1 \times 10^{15} \mathrm{n}_{\mathrm{eq}} / \mathrm{cm}^{2}$ [33]. On the contrary, it did not reproduce the charge collection properties of 3D sensors irradiated with protons at $2 \times 10^{16} \mathrm{n}_{\mathrm{eq}} / \mathrm{cm}^{2}$, and in particular it failed to predict the experimentally observed double-junction effect [27]. Improved trap models should therefore be used in the future to account for radiation damage at these very high fluences.
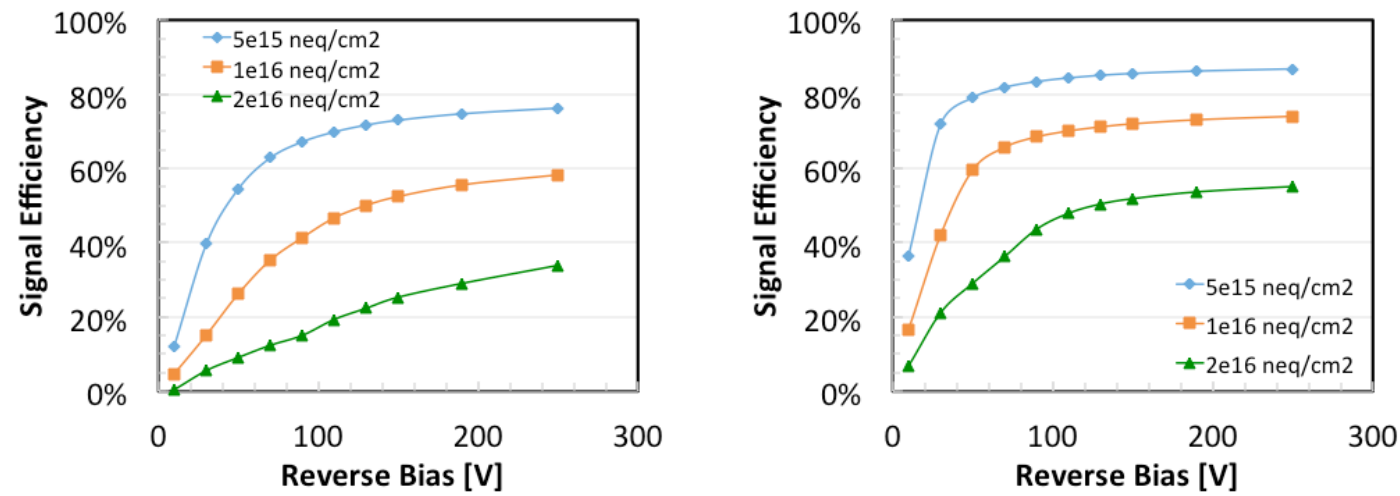

Figure 4 Simulated signal efficiency at different irradiation fluences for the two pixel layouts: $50 \times 50 \mu^{2}$ (left) and $25 \times 100 \mu^{2}$ (right).

\section{Conclusions}

Very impressive progress has been achieved in 3D detectors in the past few years, boosted by the ATLAS IBL project, and including demonstration of medium volume productions with reasonably good yield. However, the very demanding requirements of the phase 2 upgrades at HL-LHC call for the development of a new generation of 3D pixel sensors, with $\sim 2 \mathrm{x}$ downscaling of all device dimensions, and improved radiation tolerance.

A 3-year R\&D program (namely the ACTIVE project) has been jointly proposed to INFN by ATLAS and CMS to push 3D pixel technology towards HL-LHC requirements. The ATLAS-CMS synergy and the tight partnership with FBK can give a unique opportunity for technical leadership in the most demanding inner-most layers of HL-LHC detectors. During the first year of the project, FBK successfully proved the feasibility of the most critical process 
steps (e.g., the etching by DRIE and filling with poly-Si of narrow columns), and the quality of a new raw material (e.g., SiSi direct bonded wafers). In parallel, the TCAD simulations, design and layout editing of new 3D sensors have been performed at the University of Trento. The fabrication of the first 3D batch is now being started at FBK.

\section{Acknowledgements}

This work has been partially funded by: (i) the Provincia Autonoma di Trento through the Project MEMS3; (iii) the Italian National Institute for Nuclear Physics (INFN) through the Projects ATLAS, CMS, and RD_FASE2 (CSN1).

Fruitful discussions with colleagues from the INFN "ACTIVE" Collaboration and FBK are warmly acknowledged. A special thanks to Dr. Cinzia Da Vià (University of Manchester, UK) for providing the updated plot of signal efficiency, and to Dr. Roberto Mendicino (University of Trento, Italy) for performing the TCAD simulations.

\section{References}

[1] S. I. Parker, et al., 3D - A proposed new architecture for solid-state silicon detectors", Nucl. Instrum. Methods A 395 (1997) 328.

[2] S. I. Parker, et al., Increased Speed: 3D Silicon Sensors; Fast Current Amplifiers, IEEE Trans. Nucl. Sci. NS-58(2) (2011) 404.

[3] G.-F. Dalla Betta, et al., $3 D$ silicon sensors: irradiation results, in Proceedings of $21^{\text {st }}$ International Workshop on Vertex Detectors PoS(Vertex2012) 014.

[4] C.J. Kenney, et. al., Results from 3-D silicon sensors with wall electrodes: near-cell-edge sensitivity measurements as a preview of active-edge sensors, IEEE Trans. Nucl. Sci. NS-48(6) (2001) 2405.

[5] C.J. Kenney, et al., Silicon detectors with 3-D electrode arrays: fabrication and initial test results, IEEE Trans. Nucl. Sci. NS-46(4) (1999) 1224.

[6] S. H. Christiansen, et al., Wafer direct bonding: from advanced substrate engineering to future applications in micro/nanoelectronics, Proc. of the IEEE 94 (2006) 2060.

[7] B. Wu, et al., High aspect ratio silicon etch: A review, J. Applied Physics 108 (2010) 051101.

[8] C. Da Vià, et al., 3D Silicon Sensors: Design, Large Area Production and Quality Assurance for the ATLAS IBL Pixel Detector Upgrade, Nucl. Instrum. Methods A 694 (2012) 321.

[9] The ATLAS IBL Collaboration, Prototype ATLAS IBL Modules using the FE-I4A Front-End Readout Chip, JINST 7 (2012) P11010.

[10] C. Da Vià, et al., 3D active edge silicon sensors: device processing, yield and QA for the ATLASIBL production, Nucl. Instrum. Methods A 699 (2013) 18.

[11] The ATLAS Forward Detector Project, http://atlas-project-lumi-fphys.web.cern.ch/atlas-projectlumi-fphys/

[12] The ATLAS Collaboration, Letter of Intent for the Phase-II Upgrade of the ATLAS Experiment, CERN-2012-022, LHCC-I-023, 2012.

[13] S. Grinstein, et al., Beam test studies of 3D pixel sensors irradiated non-uniformly for the ATLAS forward physics detector, Nucl. Instrum. Methods A 730 (2013) 28. 
[14] T.-E. Hansen, et al., First fabrication of full 3D-detectors at SINTEF, JINST 4 (2009) P03010.

[15] G. Pellegrini, et al., First double sided 3-D detectors fabricated at CNM-IMB, Nucl. Instrum. Methods A $\mathbf{5 9 2}$ (2008) 38.

[16] A. Zoboli, et al., Double-Sided, Double-Type-Column 3D detectors at FBK: Design, Fabrication and Technology Evaluation, IEEE Trans. Nucl. Sci. NS-55(5) (2008) 2775.

[17] G. Giacomini, et al., Development of double-sided full-passing-column 3D Sensors at FBK, IEEE Trans. Nucl. Sci. vol. NS-60(3) (2013) 2357.

[18] M. Povoli, et al., Slim edges in double-sided silicon 3D detectors, JINST 7 (2012) C01015.

[19] C. Gemme, 3D sensors for tracking detectors: present and future applications", in Proceedings of $22^{\text {nd }}$ International Workshop on Vertex Detectors PoS(Vertex2013) 028.

[20] M. Povoli, et al., Layout and Process Improvements to Double-Sided Silicon 3D Detectors Fabricated at FBK, in Conference Record of 2012 IEEE Nuclear Science Symposium, N14-204.

[21] G.-F. Dalla Betta, et al., Characterization of New FBK Double-Sided 3D Sensors with Improved Breakdown Voltage, in Conference Record of 2013 IEEE Nuclear Science Symposium, N41-1.

[22] E. Alagoz, et al., Simulation and laboratory test results of $3 D$ CMS pixel detectors for HL-LHC, JINST 7 (2012) P08023.

[23] A. Kryzda, et al., Pre- and post-irradiation performance of FBK 3D silicon pixel detectors for CMS, Nucl. Instrum. Methods A 763 (2014) 404.

[24] C. Da Vià, S. Watts, The geometrical dependence of radiation hardness in planar and 3D silicon detectors, Nucl. Instrum. Methods A. 603 (2009) 319.

[25] M. Koehler, et al., Comparative measurements of highly irradiated $n$-in-p and p-in-n $3 D$ silicon strip detectors, Nucl. Instrum. Methods A. 659 (2011) 272.

[26] C. Da Vià, et al., 3D active edge silicon sensors with different electrode configurations: Radiation hardness and noise performance, Nucl. Instrum. Methods A. 604 (2009) 505.

[27] G.-F. Dalla Betta, et al., Radiation hardness tests of double-sided 3D strip sensors with passingthrough columns, Nucl. Instrum. Methods A. 765 (2014) 155.

[28] M. Garcia-Sciveres, J. Christainsen, Development of pixel readout integrated circuits for extreme rate and radiation, CERN-LHCC-2013-002, LHCC-I-024. http://rd53.web.cern.ch/RD53

[29] M. Povoli, et al., Design and testing of an innovative slim-edge termination for silicon radiation detectors, JINST 8 (2013) C11022.

[30] M. Povoli, et al., Impact of the layout on the electrical characteristics of double-sided silicon 3D sensors fabricated at FBK, Nucl. Instrum. Meth. A 699 (2013) 22.

[31] M. Petasecca, et al., Numerical Simulation of Radiation Damage Effects in p-Type and n-Type FZ Silicon Detectors, IEEE Trans. Nucl. Sci. NS-53(5) (2006) 2971.

[32] D. Pennicard, et al., Simulations of radiation-damaged 3D detectors for the Super-LHC, Nucl. Instrum. Methods A. 592 (2008) 16.

[33] A. La Rosa, et al., Characterization of proton irradiated 3D-DDTC pixel sensor prototypes fabricated at FBK, Nucl. Instrum. Methods A 681 (2012) 25. 